\title{
Comparison of two superconducting elliptical undulators for generating circularly polarized light
}

\author{
C. S. Hwang and P. H. Lin \\ NSRRC, 101 Hsin-Ann Road, Hsinchu Science Park, Hsinchu 30077, Taiwan
}

(Received 7 April 2004; published 16 September 2004)

\begin{abstract}
The potential use of two planar superconducting elliptical undulators - a vertically wound racetrack coil structure and a staggered array structure - to generate a circularly polarized hard $\mathrm{x}$-ray source was investigated. The magnetic poles and wires of the up and down magnet arrays were rotated in alternating directions on the horizontal plane, an elliptical field is generated to provide circularly polarized light in the electron-storage ring and the energy-recovery linac accelerator. Rapid switching between right- and left-circularly polarized radiations is performed using two undulators with oppositely rotated wires and poles. Given a periodic length of $15 \mathrm{~mm}$ and a gap of $5 \mathrm{~mm}$, the magnetic-flux densities in the elliptical undulator are $B_{z}=1.2 \mathrm{~T}\left(B_{x}=0.6 \mathrm{~T}\right)$ and $B_{z}=0.35 \mathrm{~T}\left(B_{x}=\right.$ $0.15 \mathrm{~T}$ ) in the planar vertically wound racetrack coil and the staggered structure with poles rotated by $35^{\circ}$ and $25^{\circ}$, respectively. In maximizing the merit of the flux and the width of the effective field region in the two superconducting elliptical undulators, the trade-off rotation angles of the coils and poles are $20^{\circ}$ and $5^{\circ}$, for vertically wound racetrack coil and staggered undulators, respectively.
\end{abstract}

DOI: 10.1103/PhysRevSTAB.7.090701

PACS numbers: 41.60.Ap, 41.85.Lc, 41.60.Cr

\section{INTRODUCTION}

Various schemes [1-5] have been applied to generate helically or elliptically distributed fields, using electromagnets with copper coils, and hybrid or pure structures with permanent magnets in various configurations. These schemes, with either helical geometry, which combines separate horizontal and vertical devices, or planar geometry with variable phase, yield variably polarized light. These schemes currently pertain to an undulator with a long period that has been installed in the low- or medium-energy storage ring, to radiate circularly polarized soft $x$ ray. A planar superconducting short-period undulator with a pure vertical field $[6,7]$ in a mediumenergy accelerator facility has been developed to provide linearly polarized hard $\mathrm{x}$ ray. However, circularpolarization (CP) experiments on inelastic magnetic scattering, spin-dependent photoabsorption, and magnetic circular dichroism in the hard $\mathrm{x}$-ray energy region must be undertaken. Hence, a planar superconducting elliptical undulator for generating circularly polarized hard $\mathrm{x}$ ray in the medium-energy accelerator is launched. Consequently, two types of planar superconducting minipole undulators - staggered and vertically wound racetrack coil structures [8] with rotated poles and wires, respectively - were studied to create circular polarization in the hard $\mathrm{x}$-ray range of the low- and mediumenergy accelerator facility. These two types of undulators that have two identical undulators with oppositely rotated poles and are associated with five kickers can switch rapidly between right- and left-circularly polarized radiations.

These two types of magnets will provide more intense circularly polarized hard $\mathrm{x}$ rays to satisfy the increasing number of users. Hence, this study discusses the elliptical field producing mechanism of the two planar superconducting undulators and the design of the magnet array structures. Meanwhile, the characteristics of the elliptical magnetic field and the photon spectra of the two elliptical undulators are investigated. The comparison between the staggered and vertically wound racetrack coil structures, including the merits of the photon flux in the circularly polarizing mechanism and the impact of the magnetic field on the electron-beam, are considered.

\section{MECHANISM FOR PRODUCING AN ELLIPTICAL FIELD}

Figures 1(a) and 1(b) present the up and down arrangement of magnetic-pole arrays employed to generate pure
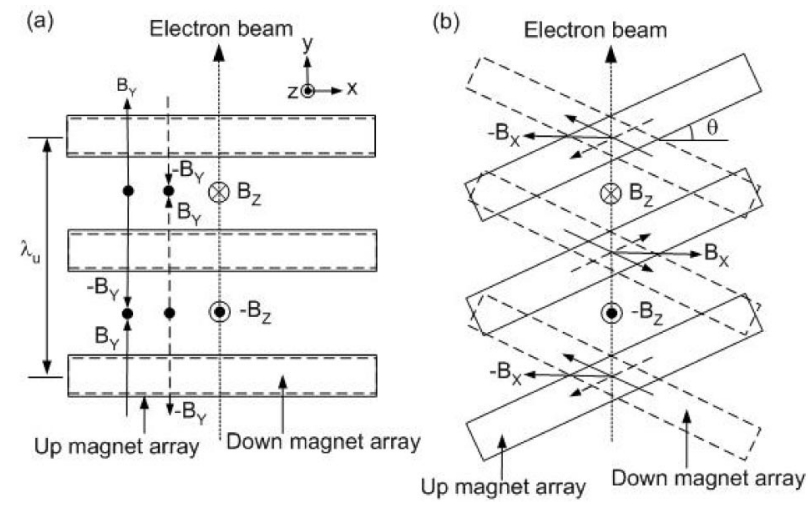

FIG. 1. Schematic top view of the magnetic-pole arrays that are (a) unrotated and (b) rotated in the horizontal plane to produce the pure vertical and the elliptical fields in the planar superconducting undulator. Dashed and solid lines represent the down and up magnet arrays, respectively. 
vertical and elliptical fields in a planar superconducting undulator, respectively $[7,8]$. The vertically wound racetrack coils replaced the permanent magnet to magnetize the iron pole. When the up and down magnetic-pole arrays are parallel, as shown in Fig. 1(a), a superposition of the two sinusoidal fields of the up and down magneticpole arrays without rotation of the wire $\left(\theta=0^{\circ}\right)$ yields the following equations (1)-(3), which fully express the magnetic-field components near the axis [9] in the racetrack coil structure:

$$
\begin{gathered}
B_{z}=B_{z o} \cosh \left(\phi_{z} z\right) \cosh \left(\phi_{x} x\right) \cos \left(\phi_{0} y\right), \\
B_{x}=-\frac{\phi_{x}}{\phi_{z}} B_{z o} \sinh \left(\phi_{z} z\right) \sinh \left(\phi_{x} x\right) \cos \left(\phi_{0} y\right), \\
B_{y}=-\frac{\phi_{0}}{\phi_{z}} B_{z o} \sinh \left(\phi_{z} z\right) \cosh \left(\phi_{x} x\right) \sin \left(\phi_{0} y\right),
\end{gathered}
$$

in which $\phi_{x}^{2}+\phi_{z}^{2}=\phi_{0}^{2}=\left(2 \pi / \lambda_{u}\right)^{2}$ and $\lambda_{u}$ represents the periodic length. An exciting current in the superconducting coil produces the $B_{z o}$. In this case, only the vertical sinusoidal field distribution $B_{z}(y)$ is evident in the midplane $(z=0)$ and the field distribution is as plotted in Fig. 2(a).

When the magnetic-pole arrays of the staggered undulator are placed in a superconducting solenoid magnet that generates a longitudinal field $B_{o}$ to magnetize the poles, the magnetic fields become as specified by (4)-(6),

$$
B_{z}=B_{z o} \cosh \left(\phi_{z} z\right) \cosh \left(\phi_{x} x\right) \cos \left(\phi_{0} y\right),
$$
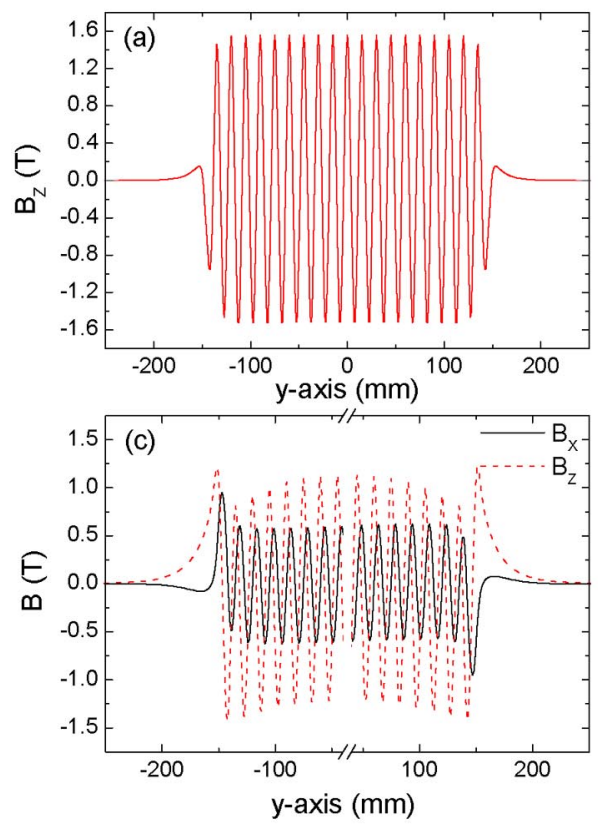

$$
\begin{array}{r}
B_{x}=-\frac{\phi_{x}}{\phi_{z}} B_{z o} \sinh \left(\phi_{z} z\right) \sinh \left(\phi_{x} x\right) \cos \left(\phi_{0} y\right), \\
B_{y}=B_{0}-\frac{\phi_{0}}{\phi_{z}} B_{z o} \sinh \left(\phi_{z} z\right) \cosh \left(\phi_{x} x\right) \sin \left(\phi_{0} y\right),
\end{array}
$$

in which $B_{z o}=\left[2 B_{0} \sin (\pi f)\right] /\left[\sinh \left(\pi g / \lambda_{u}\right) \pi f\right]$ was produced by the solenoid longitudinal field $B_{o}$ and $g$ and $f$ represent the magnet gap and the ratio of the width of the spacer to the period of the staggered undulator [10], respectively. In this case, only the vertical sinusoidal field distribution $B_{z}(y)$ pertains in the midplane $(z=0)$ and the field distribution is as indicated in Fig. 2(b). Figure 3 reveals that the longitudinal field $B_{o}$ of the superconducting solenoid magnet determines the vertical field strength $B_{z}$. The maximum field strength $B_{z}$ is obtained at the longitudinal field $B_{o}=0.87 \mathrm{~T}$.

If the up and down magnetic-pole arrays of the planar undulator are rotated by an angle $\theta$ in the horizontal plane, as shown in Fig. 1(b), then the elliptical fields $B_{x}$ and $B_{z}$ in the midplane $(z=0)$ and along the longitudinal axis are generated simultaneously, but with a phase difference of $\pi / 2$; here, $\theta$ is the angle between the directions of the rotated and the unrotated magnetic poles on the transverse $x$ axis. Figures 4(a) and 4(b) present a threedimensional view of the rotation of the magnetic-pole arrays in the horizontal plane. The on-axis $(x=z=0)$ elliptical field on the rotated magnetic-pole arrays of the planar superconducting undulator in the racetrack coil structure and the staggered structure are expressed ap-
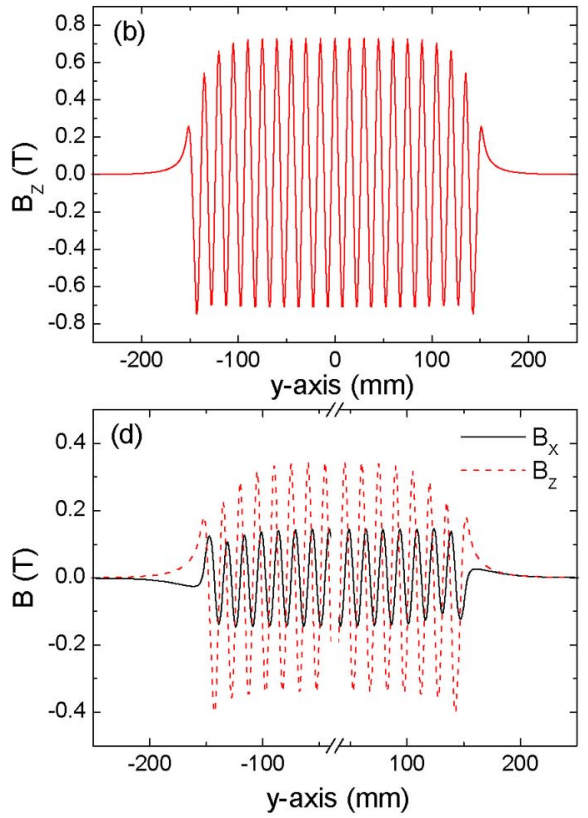

FIG. 2. (Color) Distribution of magnetic-flux density along the longitudinal $y$ axis of the linearly polarized undulator in (a) the racetrack coil structure, (b) the staggered structure, and the elliptically polarized undulator in (c) the racetrack coil structure and (d) the staggered structure. The selected angles of rotation of the vertically wound racetrack coil and staggered undulator are $35^{\circ}$ and $25^{\circ}$. 


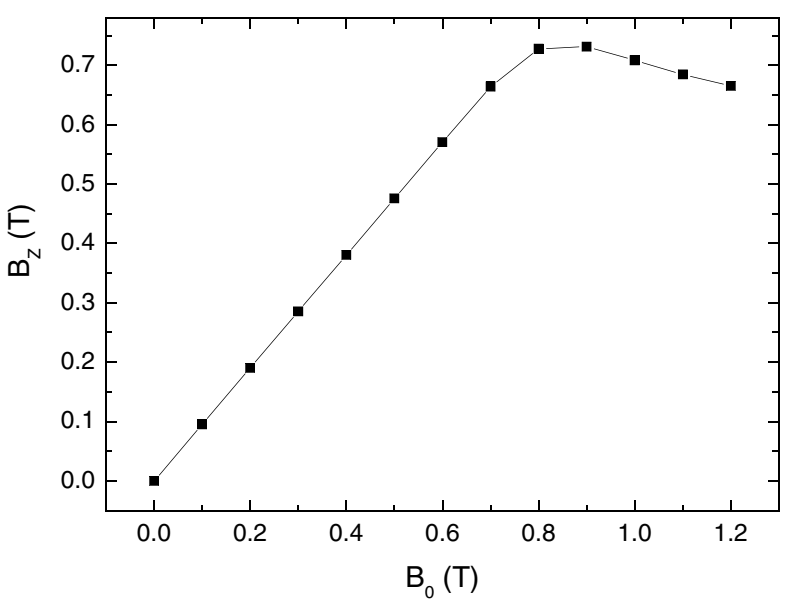

FIG. 3. Maximum vertical field strength $B_{z}$ as a function of the longitudinal field $B_{o}$ that was generated by the superconducting solenoid magnet.

proximately by (7) [11],

$$
\begin{aligned}
& B_{z}(y)=B_{z o} \cos \left(2 \pi y / \lambda_{u}\right), \\
& B_{x}(y)=B_{y} \sin \theta=-\frac{\phi_{0}}{\phi_{z}} B_{z o} \sin \left(2 \pi y / \lambda_{u}\right) \sin \theta .
\end{aligned}
$$

Therefore, the elliptical fields of $B_{x}$ and $B_{z}$ are out of phase and so generate elliptically polarized radiation. Figures 2(c) and 2(d) depict the elliptical field distributions of $B_{x}$ and $B_{z}$. Two equal elliptical undulators with oppositely rotated wires and poles (one rotated by an angle $\theta$ and the other rotated by an angle $-\theta$ ) should be installed in the same straight section and operated with five cooperating kickers [12]. Consequently, this mechanism will execute rapid switching between right and left circular polarizations.

(a)

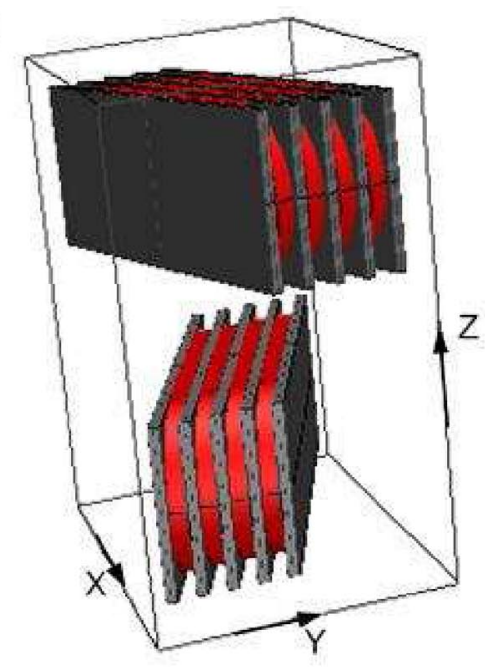

Table I lists the main parameters that govern the design of the magnet and the spectral characteristics of both types of superconducting undulators. Figures 5(a) and 5(b) plot the horizontal and vertical magnetic-flux densities as functions of the angle $\theta$ of rotation of the poles in the racetrack coil and the staggered structure undulator, respectively. The vertical field $B_{z}$ of the vertically wound racetrack coil and the staggered structure decreases as the rotation angle $\theta$ is increased. However, the horizontal field $B_{x}$ increases, and then decreases, as the rotation angle $\theta$ is increased. Increasing the angle $\theta$ from $0^{\circ}$ to $35^{\circ}$ and $25^{\circ}$ maximizes the magnetic-flux densities $B_{x}$ to 0.6 and $0.15 \mathrm{~T}$ in the racetrack coil and the staggered undulator, respectively. However, the maximum magnetic-flux densities $B_{z}$ decrease from 1.55 and $0.72 \mathrm{~T}$ to 1.2 and $0.35 \mathrm{~T}$, respectively.

\section{PROPERTIES OF ELLIPTICAL MAGNETIC FIELD}

The field homogeneity and the distribution of the integral field on the transverse axis were examined to investigate the effect of the magnetic field on the electron beam. The homogeneity of the magnetic field should be sufficiently broad to tolerate small errors in the spectral phase and magnet alignment, and improve the photon coherence condition. Figures 6(a) and 6(b) plot the calculated field deviation $\Delta B / B$ and demonstrate that the rolloff $\Delta B / B$ falls rapidly as the rotation angle of the magnetic poles and coil arrays increases. The homogeneity of the field on the two transverse axes of the vertically wound racetrack coil undulator reveals that the rotation angle is as small as possible. Figures 6(c) and 6(d) plot the asymmetrical field distribution of the roll-off $\Delta B / B$ with rotated poles in the staggered undulator. Therefore, the

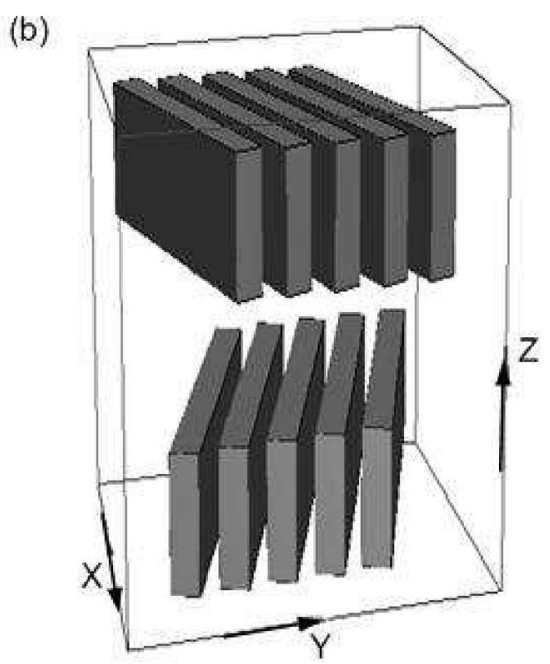

FIG. 4. (Color) Three-dimensional views of the elliptically polarized undulators $(\theta \neq 0)$ in (a) the racetrack coil structure (whose whole iron yoke was machined into many grooves for coil winding, with an angle $\theta$ between the transverse direction of the iron yoke and the groove) and (b) the staggered structure. 
TABLE I. Main spectral features and parameters that govern the design of the superconducting elliptical undulator in the $1.5 \mathrm{GeV}$ storage ring.

\begin{tabular}{lcc}
\hline \hline & Racetrack coil & Staggered \\
\hline Number of poles $N$ & 121 & 121 \\
Periodic length $\lambda_{u}(\mathrm{~cm})$ & 1.5 & 1.5 \\
Magnetic gap $g(\mathrm{~mm})$ & 5 & 5 \\
Physical length $L(\mathrm{~cm})$ & 93 & 93 \\
Rotation angle $\theta(\mathrm{deg})$ & 20 & 5 \\
Solenoid longitudinal field $B_{0}(\mathrm{~T})$ & 0 & 0.87 \\
Coil current density $\left(\mathrm{A} / \mathrm{mm}^{2}\right)$ & 900 & 34 \\
Magnetic-flux density $B_{z}\left(B_{x}\right)(\mathrm{T})$ & $1.36(0.46)$ & $0.65(0.05)$ \\
Deflection parameter $K_{z}\left(K_{x}\right)$ & $1.9(0.64)$ & $0.91(0.07)$ \\
Electron wiggler angle $\theta_{z}\left(\theta_{x}\right)(\mathrm{mrad})$ & $0.22(0.65)$ & $0.02(0.31)$ \\
Electron wiggler amplitude $z(x)(\mu \mathrm{m})$ & $0.52(1.55)$ & $0.05(0.74)$ \\
Photon energy $E_{p}(\mathrm{CP})(\mathrm{keV})$ & $0.5-6$ & $1-1.3(1 \mathrm{st}$ harmonic) \\
Half-width radiation cone at $1 \mathrm{st}$ harmonic $\phi_{z}\left(\phi_{x}\right)(\mu \mathrm{rad})$ & $34(52)$ & $31(37)$ \\
Circular-polarization rate & $0.87-0.93$ & $0.45-0.6$ \\
Merit flux for 9 th harmonic $[\mathrm{photon} /(\mathrm{s} 0.1 \%$ bw $0.2 \mathrm{~A})]$ & $4 \times 10^{13}$ & $9 \times 10^{10}$ \\
\hline \hline
\end{tabular}

quadrupole and higher harmonic components are intrinsically strong when the poles are rotated in the staggered undulator. Figures 6(c) and 6(d) also indicate that the difference between the roll-off range at rotation angle $5^{\circ}$ and that at $20^{\circ}$ is not obvious. The field homo-

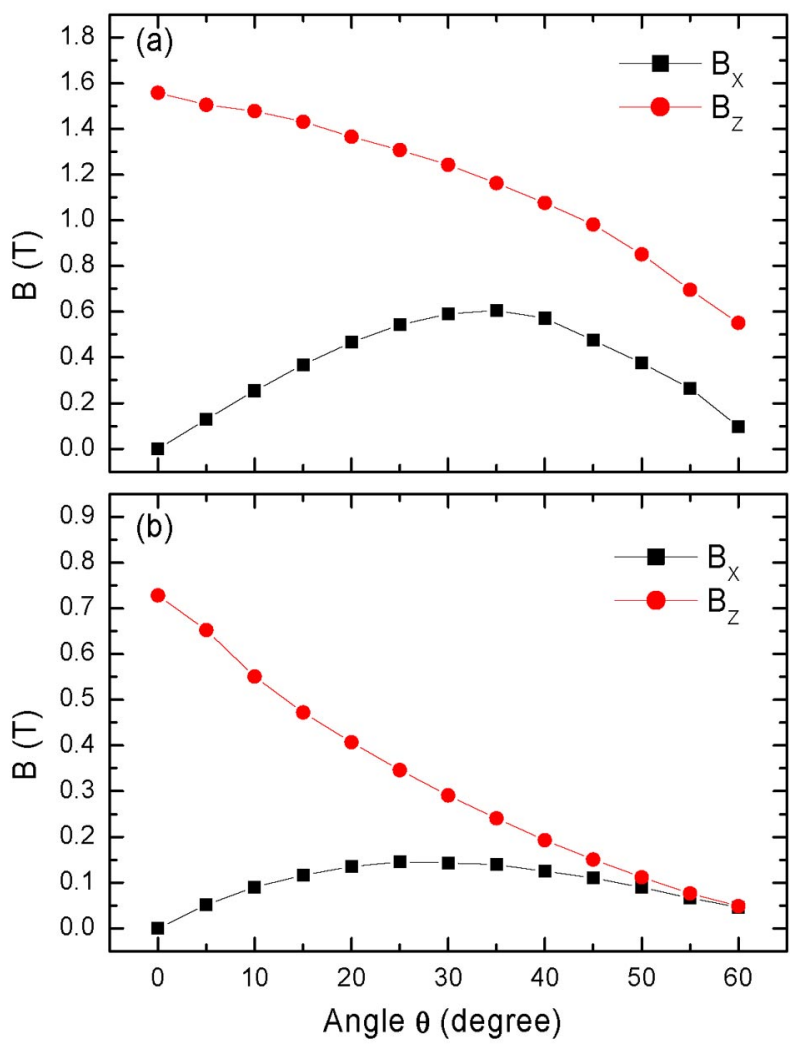

FIG. 5. (Color) Magnetic-flux densities $B_{z}$ and $B_{x}$ as functions of rotation angle $\theta$ in (a) the racetrack coil structure and (b) the staggered structure. The period was fixed at $1.5 \mathrm{~cm}$. geneity of the vertically wound racetrack coil undulator is much better than that of the staggered undulator under elliptical field conditions. Table II shows that the typical range of the roll-off $\Delta B / B$ is kept at $0.2 \%$ on the transverse axis.

The effective region of the integral field should be sufficiently large to maintain a large dynamic aperture to meet the dynamic requirements of the electron beam. Figure 7 plots the calculated effective region of the integral field $\int B(x, y) d y$ on the horizontal and vertical axes. Figures 7(a) and 7(b) demonstrate that the multipole components become stronger than those obtained without rotation of the wires and poles. Hence, the angle of rotation of the vertically wound racetrack coil undulator should be as small as practicable. The integrated vertical field distribution on the transverse axis is symmetrical and the integrated strength of the horizontal field distribution in the racetrack coil undulator is very small. Figures 7(c) and 7(d) indicate that the poles were rotated in the staggered undulator, producing strong variations of the integrated vertical field along the $x$ axis and the integrated horizontal field along the $z$ axis. Meanwhile, the integral multipole field strengths of the vertically wound racetrack coil undulator were smaller than those of the staggered undulator. The strengths of the multipole field components were calculated for the wires and poles rotated through $20^{\circ}$ and $5^{\circ}$ in the racetrack coil and the staggered undulator, respectively, as shown in Table II. A 12-pole magnet with quadrupole, sextupole, octupole, decapole, and dodecapole fields is required to compensate for the multipole components, especially in the rotated staggered undulator.

Furthermore, the longitudinal field $B_{o}$ in the staggered undulator will perturb the elliptical trajectories. The Lorentz force $\vec{F}_{x}=m \ddot{x}=q \vec{v}_{y} \times \vec{B}_{z}$ will guide the elec- 

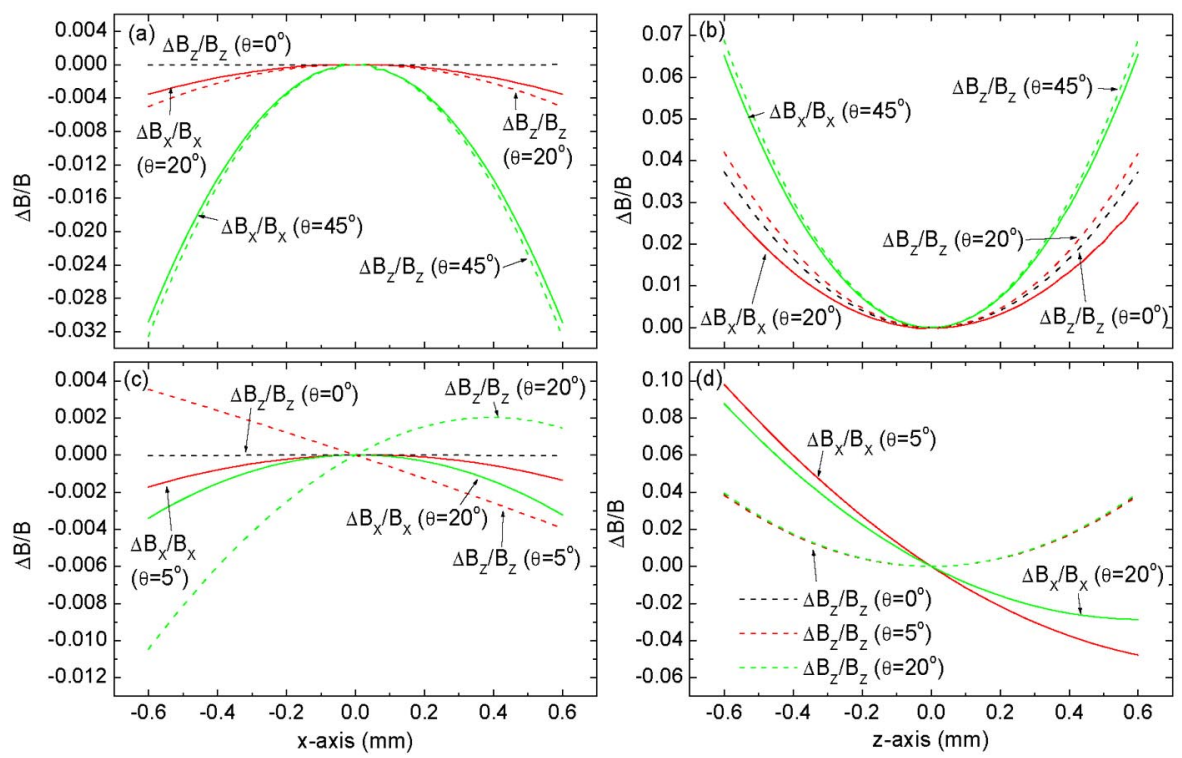

FIG. 6. (Color) Roll-off $\Delta B / B$ as functions of distance along the transverse axis: (a) and (b) field homogeneity of $\Delta B_{x}(x, z) / B_{x}(0,0)$ and $\Delta B_{z}(x, z) / B_{z}(0,0)$ of the racetrack coil undulator and (c) and (d) of the staggered undulator.

TABLE II. Magnetic field features of a superconducting elliptical undulator in a racetrack coil and staggered undulators in which wires and poles are rotated by $20^{\circ}$ and $5^{\circ}$, respectively; the multipole components were analyzed on the transverse $x$ axis \pm 10 mm.

\begin{tabular}{lcc}
\hline \hline & Racetrack coil & Staggered \\
\hline Roll-off range at $\Delta B_{z} / B_{z} \leq 0.2 \%(\mathrm{~mm})$ & $x= \pm 0.4, z= \pm 0.2$ & $x= \pm 0.3, z= \pm 0.14$ \\
Roll-off range at $\Delta B_{x} / B_{x} \leq 0.2 \%(\mathrm{~mm})$ & $x= \pm 0.5, z= \pm 0.2$ & $x= \pm 0.7, z= \pm 0.02$ \\
Normal (skew) quadrupole $(\mathrm{G})$ & $-5(9)$ & $-19859(0.3)$ \\
Normal (skew) sextupole $(\mathrm{G} \mathrm{cm})$ & $-11978(-1)$ & $-660(-0.03)$ \\
Normal (skew) octupole $\left(\mathrm{G} \mathrm{cm}^{2}\right)$ & $2(10)$ & $-17664(0.2)$ \\
Normal (skew) decapole $\left(\mathrm{G} \mathrm{cm}^{3}\right)$ & $60349(-0.2)$ & $-15(-0.2)$ \\
\hline \hline
\end{tabular}

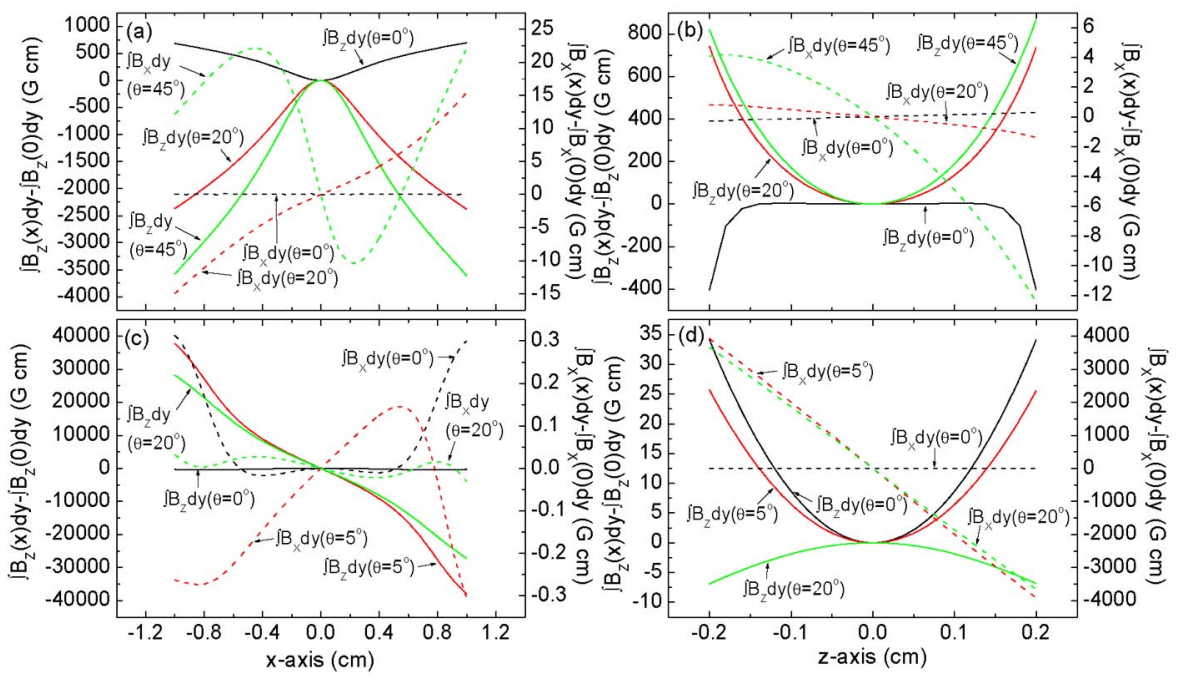

FIG. 7. (Color) Distribution of calculated integral field strength along the transverse axis: the region of the effective field that includes $\int B_{x}(x, z) d y$ and $\int B_{z}(x, z) d y$ in (a) and (b) the racetrack coil undulator and in (c) and (d) the staggered undulator. 
tron trajectories in the transverse plane, that is approximately expressed as (8)

$$
x=\frac{K_{z} \lambda_{u}}{2 \pi \gamma} \sin \left(2 \pi y / \lambda_{u}\right), \quad z=\frac{K_{x} \lambda_{u}}{2 \pi \gamma} \cos \left(2 \pi y / \lambda_{u}\right),
$$

in which $K_{z, x}=0.934 B_{z, x} \lambda_{u}$, is the deflection value, and $B_{z, x}$ and $\lambda_{u}$ are measured in units of $\mathrm{T}$ and $\mathrm{cm}$, respectively. Table I also lists the maximum wiggler amplitudes along the two transverse axes. Hence, the elliptical planar undulator of the vertically wound racetrack coil structure produces pure elliptical trajectories in the transverse plane. However, an intrinsic strong longitudinal field $B_{o}$ in the staggered undulator disturbs the elliptical trajectories in the two transverse axes of the $x-z$ plane. The longitudinal field $B_{o}$ perturbed the electron trajectories; the perturbations were around 0.27 and $0.02 \mathrm{~nm}$ on the vertical and horizontal transverse axes, respectively.
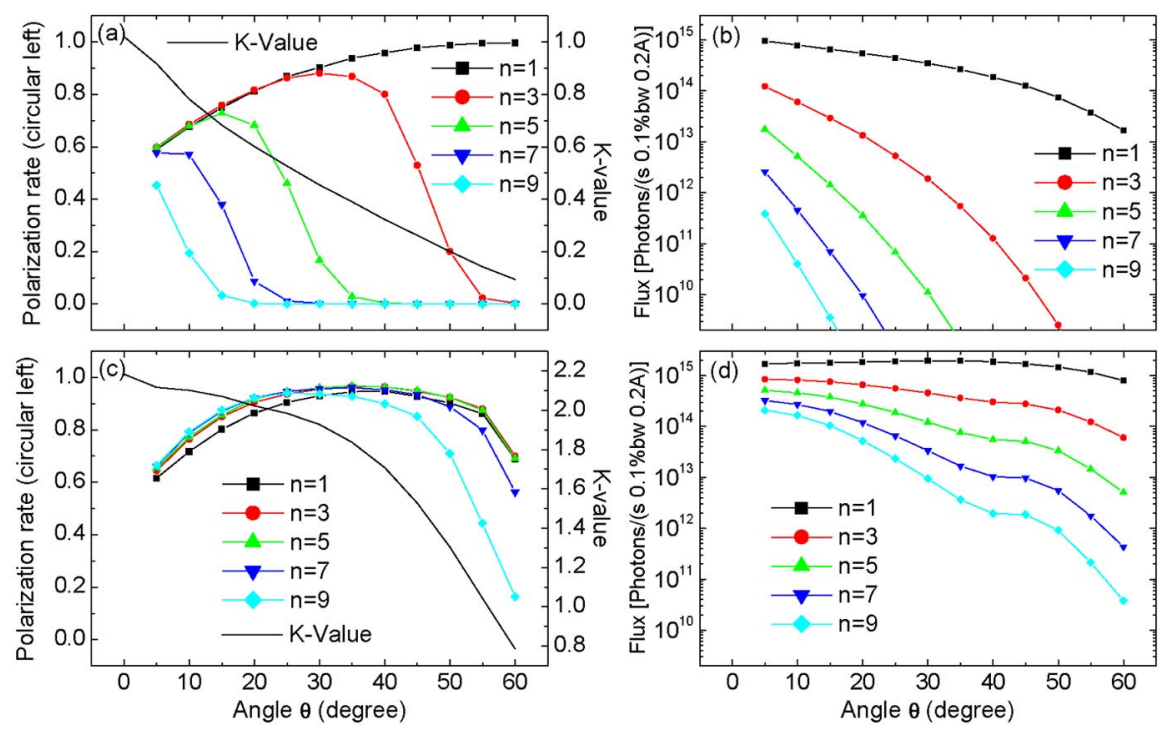

FIG. 8. (Color) Polarization rate and photon energy as functions of rotation angle $\theta$ for (a) and (b) the staggered undulator, whose photon flux is calculated at the maximum deflection $K$ at each rotation angle with $\lambda_{u}=1.5 \mathrm{~cm}$, and for (c) and (d) the racetrack coil undulator, whose photon flux is calculated at the maximum deflection value $K$ at each rotation angle with $\lambda_{u}=1.5 \mathrm{~cm}$. The deflection parameter is $K=\sqrt{K_{x}^{2}+K_{z}^{2}}$.
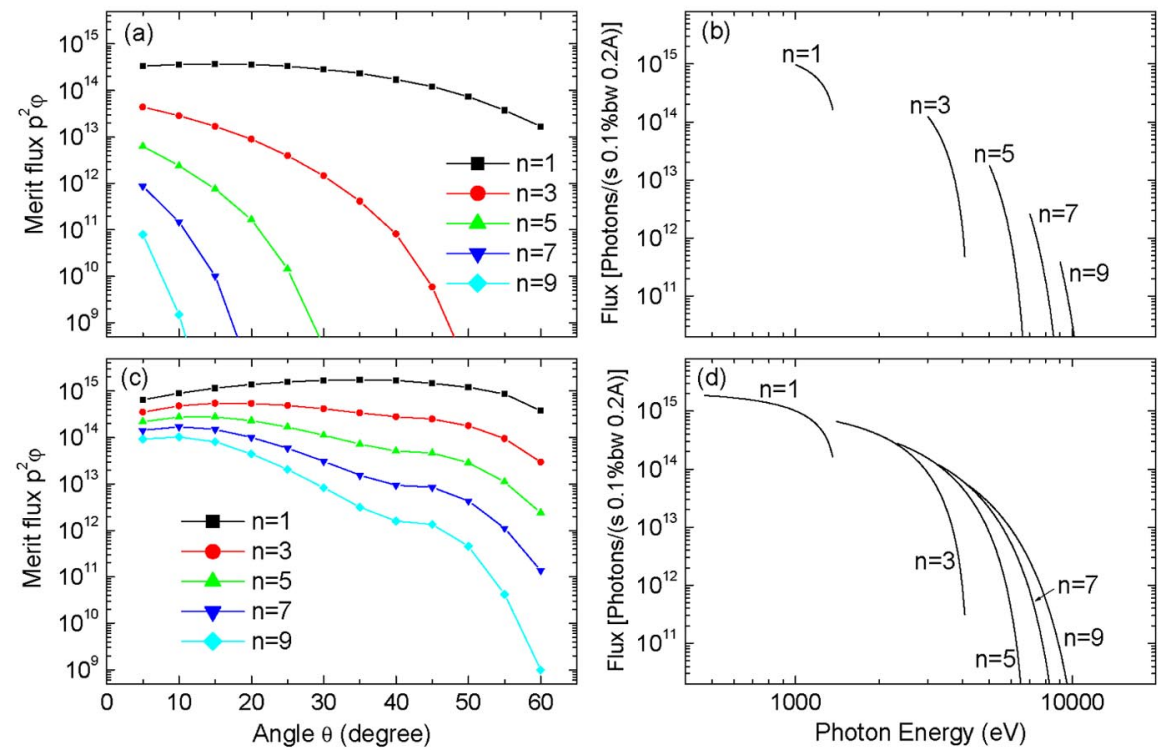

FIG. 9. (Color) Merit flux as functions of rotation angle $\theta$, and the spectrum of the photon flux, for (a) and (b) the staggered undulator, with the photon flux in (b) calculated at $\theta=5^{\circ}$, and for (c) and (d) the racetrack coil undulator, with the photon flux in (d) calculated at $\theta=20^{\circ}$. The spectrum was calculated at an electron energy of $1.5 \mathrm{GeV}$ and an electron current of $200 \mathrm{~mA}$. 


\section{MERIT FLUX ANALYSIS}

Figure 8 shows the rate of polarization and the photon flux of the two types of elliptical undulators. These values are calculated at a natural emittance of $21.4 \mathrm{~nm}$ rad and energy spread of $0.2 \%$. Evidently, the polarization rate of each spectral harmonic in the vertically wound racetrack coil undulator increases and then decreases as the angle of rotation is increased [Fig. 8(c)]. The polarization rate of each harmonic spectrum is approximately 0.92 when the wires are rotated to $35^{\circ}$. However, the polarization rate of the higher harmonic spectrum of the staggered undulator [Fig. 8(a)] does not close to 1 as the rotation angle is increased. Figure 8(b) reveals that the photon flux of each harmonic spectrum falls rapidly as the angle of rotation of the poles increases. Therefore, the polarization rate and photon flux of the vertically wound racetrack coil undulator clearly exceed those of the staggered undulator. Figures 9(a) and 9(c) plot the merit flux $P^{2} \varphi$ [13] as a function of angle $\theta$ for the two types of undulators; herein, $P$ is the rate of circular polarization [9] and $\varphi$
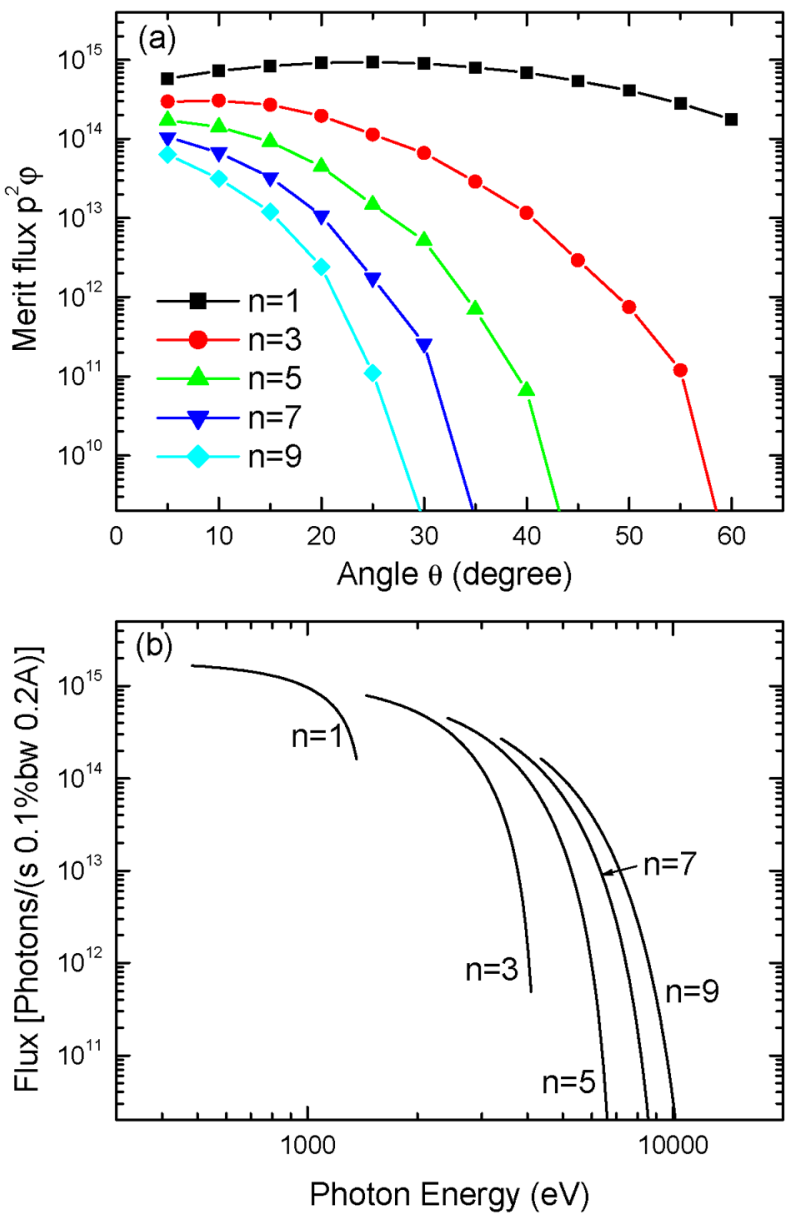

FIG. 10. (Color) (a) Merit flux as a function of the rotation angle $\theta$ of the poles in the staggered undulator and (b) spectrum of each harmonic of the staggered undulator with the poles rotated by $5^{\circ}$. The results were calculated at a magnetic gap of $2 \mathrm{~mm}$ in the staggered undulator. [photon/(s 0.1\%bw $0.2 \mathrm{~A})$ ] presents the flux of the elliptical undulator in the $1.5-\mathrm{GeV}$ electron-beam accelerator. The merit flux is maximal at rotation angles of $35^{\circ}$ and $15^{\circ}$ in the first harmonic spectra of the racetrack coil structure and the staggered structure, respectively. The optimized merit flux of the racetrack coil structure greatly exceeds that of the staggered undulator structure, at a particular angle of rotation, especially in the higher harmonic spectra.

If the homogeneity of the magnetic field affects the coherence condition and reduces the dynamic aperture of the beam, then angles of rotation $20^{\circ}$ and $5^{\circ}$ of the racetrack coil and the staggered undulator, respectively, are adopted to yield a favorable compromise among the merit fluxes of all harmonic spectra and the effect of the magnetic field on the electron beam. Figures 9(b) and 9(d) plot the calculated circularly polarized flux of the vertically wound racetrack coil and the staggered structures for which the wires and poles were rotated by $20^{\circ}$ and $5^{\circ}$, respectively. The photon energy $E_{n}$ depends on the periodic length $\lambda_{u}(\mathrm{~cm})$, when $E_{n}(\mathrm{keV})=0.95 n E^{2} /\left[\lambda_{u}(1+\right.$ $\left.\left.K^{2} / 2\right)\right]$, in which $n, E(\mathrm{GeV})$ denotes the $n$-odd harmonic numbers of the spectra and the energy of the electron beam, respectively. Figures 9(b) and 9(d) indicate that the energy spectrum of the racetrack coil undulator is continuous, but the staggered undulator gives a discontinuous spectrum. In this short-period undulator, the energy of each harmonic of the staggered undulator is not sufficiently wide to cover the entire spectrum, except when the magnet gap is maintained at $2 \mathrm{~mm}$. Figure 10(a) plots the merit flux as a function of the rotation angle of the poles, when the magnet gap of the staggered undulator is reduced to $2 \mathrm{~mm}$. Figure 10(b) shows the continuous spectrum of each harmonic of the staggered undulator, under this condition, when the poles are rotated by $5^{\circ}$, and the spectrum covers a wide range of energies.

\section{CONCLUSION}

Creating an elliptical field on a superconducting planar undulator to generate circularly polarized synchrotron light poses no great problem. The compromise result demonstrates that the circular polarization of the racetrack coil undulator is more efficient than that of the staggered undulator. It also reveals that the optimized angles of rotation of the wire and pole are $20^{\circ}$ and $5^{\circ}$, respectively. The field homogeneity and the maximum field strength of the vertically wound racetrack coil structure are better than those of the staggered structure under the optimized elliptical polarization condition. Meanwhile, the good field region of the integral field in the vertically wound racetrack coil structure is larger than that of the staggered structure. The vertically wound racetrack coil structure with a $5 \mathrm{~mm}$ magnet gap will be enough to generate circularly polarized hard $\mathrm{x}$ rays in a medium-energy accelerator (around $3 \mathrm{GeV}$ ), but the stag- 
gered undulator should reduce the magnet gap to $2 \mathrm{~mm}$ to fit the same energy range.

\section{ACKNOWLEDGMENTS}

The authors thank the National Science Council of Taiwan for supporting this research under Contract No. NSC92-2112-M-213-019. Discussions with Professor S. Y. Lee (Indiana University), H. Wiedemann (SLAC), and Mr. C. H. Chang (NSRRC) of the concept and characteristics of the elliptical undulator are particularly appreciated. The assistance of Ms. W. P. Li in processing data and drawing is appreciated.

[1] K. Halback, Nucl. Instrum. Methods Phys. Res., Sect. A 187, 109 (1981).

[2] H. Onuki, Nucl. Instrum. Methods Phys. Res., Sect. A 246, 94 (1986).

[3] J. P. Blewett and R. Chasman, J. Appl. Phys., 48, 2692 (1977).

[4] S. Sasaki, Nucl. Instrum. Methods Phys. Res., Sect. A 347, 83 (1994).
[5] R. P. Walker and B. Diviacco, Rev. Sci. Instrum. 63, 332 (1992).

[6] Andreas Geisler, Achim Hobl, Detlef Krischel, Robert Rossmanith, and Michael Schillo, IEEE Trans. Appl. Supercond., 13, 1217 (2003).

[7] C.S. Hwang, W. P. Li, P. H. Lin, and C. H. Chang, IEEE Trans. Appl. Supercond. 14, 580 (2004).

[8] S. Sasaki, in Proceedings of the Workshop on Superconducting Undulators and Wigglers, Grenoble, 2003 (ESRF, Grenoble, 2003).

[9] R. P. Walker, CERN Report No. CERN 95-06, 1995, Vol. II, p. 814.

[10] Y. C. Huang, H. C. Wang, R. H. Pantell, J. Feinstein, and J. Harris, Nucl. Instrum. Methods Phys. Res., Sect. A 341, 431 (1994).

[11] C. S. Hwang, C. H. Chang, T. C. Fan, F. Y. Lin, Ch. Wang, Shuting Yeh, H. P. Chang, K. T. Hsu, L. H. Chang, P. K. Tseng, and T. M. Uen, Nucl. Instrum. Methods Phys. Res., Sect. A 399, 463 (1997).

[12] T. Hara, K. Shirasawa, M. Takeuchi, T. Seike, Y. Saito, T. Muro, and H. Kitamura, Nucl. Instrum. Methods Phys. Res., Sect. A 498, 496 (2003).

[13] C.S. Hwang and S. Yeh, Nucl. Instrum. Methods Phys. Res., Sect. A 420, 29 (1999). 